\title{
Riesgos asociados al uso de Expansión Rápida del Maxilar
} Risks associated with the use of Maxillary Rapid Expansion

\author{
A. Pérez-Flores*, F. Gallegos-Delgado**, M. J. Hernández-Carrera**, \\ P. Torres-González***, P. Cuevas-Drago****, C. Fierro-Monti*****
}

\section{RESUMEN}

Introducción: Las anomalías dentomaxilares corresponden a un grupo de alteraciones que dificultan el desarrollo armónico de los maxilares, donde encontramos las anomalías transversales. La expansión rápida del maxilar (ERM) se utiliza como tratamiento de anomalías transversales, su objetivo es aumentar la distancia transversal por la separación de ambas hemiarcadas a nivel de la sutura palatina, utilizando un aparato expansor fijo y rígido. Los disyuntores más utilizados son los expansores Hass, Hyrax y de McNamara.

Objetivo: El objetivo de la presente revisión es identificar los riesgos de la RME reportados en la literatura relacionados con disyuntores Hass, Hyrax y Mc Namara.

Metodología: Se realizó una búsqueda en PubMed, Scielo, Science Direct, Trip Database y Google Académico utilizando los términos: "Rapid Maxillary Expansion", "Risks", "Child", "Maxillary Expansion", "RME", "Palatal Expansion", "Hyrax", "Hass". Se incluyeron estudios en inglés y español, sin límites en el año de publicación o tipo de estudio. Como criterios de exclusión consideramos la expansión quirúrgica, expansión lenta u otro tratamiento simultáneo durante la fase de expansión activa.

Resultados: De los artículos encontrados, veinte cumplieron los criterios de inclusión. Dentro de los riesgos se encuentran: deglución accidental del dispositivo de activación del expansor, bacteremia transitoria, desviación del septum nasal, reabsorción radicular, compromiso periodontal, cambio posicional de los cóndilos, cambios faciales de tejido blando y cambios esqueletales.

Conclusión: La ERM es un procedimiento ortopédico eficaz, sus indicaciones en el tratamiento precoz han aumentado debido a resultados positivos. De acuerdo con la bibliografía encontrada los beneficios superan considerablemente a los efectos negativos.

PALABRAS CLAVE: Expansión rapida del maxilar, riesgos, expansión palatina.
ABSTRACT
Introduction: The dentomaxillary anomalies correspond to a group of alterations that hinder the harmonic develop- ment of the jaws, where we find the transversal anomalies. Rapid Maxillary Expansion (RME) is used as a treatment for transverse anomalies, its objective is to increase the transversal distance by separating both hemiarchies at the level of the palatal suture, using a fixed and rigid expander. The most used are the Hass, Hyrax and McNamara expanders. Objective: The objective of this review is to identify the EMR risks reported in the literature related to Hass, Hyrax and Mc Namara expander.
Methodology: A search was made in PubMed, Scielo, Science Direct, Trip Database and Google Scholar using the
* Directora Diplomado Ortodoncia Interceptiva, Facultad de Odontología, Universidad de Concepción, Concepción, Chile
** Instituto de Odontoestomatología, Facultad de Odontología, Universidad Austral de Chile, Valdivia, Chile.
*** Especialidad Odontopediatría, Universidad de Valparaíso, Valparaíso, Chile.
**** Clínica Leufu, Lebu, Chile
***** Facultad de Odontología, Universidad de Concepción, Concepción, Chile. 
terms: "Rapid Maxillary Expansion", "Risks", "Child", "Maxillary Expansion", "RME", "Palatal Expansion", "Hyrax", "Hass". Studies in English and Spanish were included, without limits in the year of publication or type of study. As exclusion criteria, we consider surgical expansion, slow expansion or other simultaneous treatment during the active expansion phase.

Results: Of the articles found, twenty met the inclusion criteria. Among the risks are: accidental swallowing of the expander activation device, transient bacteremia, deviation of the nasal septum, root resorption, periodontal involvement, positional change of the condyles, soft tissue facial changes and skeletal changes.

Conclusion: $\mathrm{RME}$ is an effective orthopedic procedure, its indications in early treatment have increased due to positive results. According to the bibliography found, the benefits considerably outweigh the negative effects.

KEY WORDS: Rapid Maxillary Expansion, Risks, Expansion.

Fecha de recepción: 8 de agosto de 2019

Fecha de aceptación: 5 de septiembre de 2019

A. Pérez-Flores, F. Gallegos-Delgado, M. J. Hernández-Carrera, P. Torres-González, P. Cuevas-Drago, C. Fierro-Mon-

ti. Riesgos asociados al uso de Expansión Rápida del Maxilar. 2020; 36, (1): 21-26.

\section{INTRODUCCIÓN}

Las anomalías dentomaxilares (ADM) corresponden a un grupo de alteraciones que dificultan el desarrollo armónico de los maxilares, y se presentan simultáneamente con malposiciones dentarias, las cuales afectan la forma, función y estética del sistema estomatognático. ${ }^{(1)}$

Debido a su alta prevalencia, son consideradas un problema de salud pública, afectando a mas de un $35 \%$ de los niños de seis años, y a más del $50 \%$ de los niños de doce años. Las cuales no han mostrado una disminución significativa de sus valores en el transcurso del tiempo, a diferencia de las caries y enfermedad periodontal. ${ }^{(2)}$

La expansión rápida del maxilar (ERM) ha sido recomendada y utilizada como tratamiento de anomalías transversales del maxilar superior durante mucho tiempo, cobrando popularidad durante los últimos 25 años. ${ }^{(3)}$ Este tratamiento pretende aumentar la distancia transversal por separación de ambas hemiarcadas a nivel de la sutura media del paladar, utilizando un aparato expansor fijo y rígido, que se activa diariamente aproximadamente medio milimetro y que aumenta la base apical y el espacio disponible para las piezas dentarias permanentes. ${ }^{(3)}$. La edad ideal para su utilización es, previo a la consolidación de la sutura palatina, antes de los 12 años, para conseguir un mayor efecto terapéutico. ${ }^{(4)}$
Los disyuntores más utilizados son los expansores de tipo Hass, Hyrax y de McNamara o adhesión directa. El expansor de Hyrax y Hass se utilizan en denticiones mixtas o permanentes tempranas, compuestos por bandas cementadas a los molares y alambres que recorren las caras palatrinas de los ectores laterales. En cambio, el de McNamara se utiliza frecuentemente en etapas de dentición temporal y dentición mixta temprana. ${ }^{(5)}$ Este disyuntor está formado por una férula de acrílico adaptado a las caras oclusales de los primeros molares y premolares o dentición temporal superior, y un tornillo de expansión central al centro de la bóveda palatina. ${ }^{(4)}$

Una de las ventajas al utilizar este tipo de aparatos, es que cuando coexisten otras anomalías además de la transversal, se utiliza al disyuntor como anclaje y como parte de un tratamiento ortopédico integral. ${ }^{(5)} \mathrm{Al} \mathrm{mismo}$ tiempo, al ser fijos, no requieren de una colaboración directa por parte del paciente, a diferencia de los aparatos removibles. No obstante, existen efectos adversos y riesgos asociados a esta terapia ortopédica que no son conocidos por todos los profesionales. ${ }^{(3)}$

El objetivo de la presente revisión literaria es identificar los riesgos reportados en la literatura relacionados a la ERM realizada con disyuntores Hass, Hyrax y Mc Namara.

\section{MÉTODOS}


Se realizó una búsqueda en bases de datos electrónicas tales como: PubMed, Scielo, Science Direct, Trip Database y Google Académico. Utilizando los conceptos: "Rapid Maxillary Expansion", "Risks", "Child", "Maxillary Expansion", "RME", "Palatal Expansion", "Hyrax", "Hass".

Como criterios de inclusión en la búsqueda se incluyeron estudios en inglés y español, sin límites en el año de publicación o tipo de estudio. Como criterios de exclusión consideramos la expansión quirúrgica, expansión lenta u otro tratamiento simultáneo durante la fase de expansión activa.

Veinte artículos cumplieron con los criterios de inclusión y exclusión. De estos, uno corresponde a un caso clínico, seis a estudios longitudinales, tres a estudios clínicos, cinco a revisiones sistemáticas, cuatro a revisiones bibliográficas y un metaanálisis; publicados entre el año 1997 y 2018.

\section{CONTENIDO}

Dentro de los posibles efectos adversos que deben considerarse al momento de realizar ERM se encuentran:

\section{DEgLUCIÓN ACCIDENTAL DEL DISPOSITIVO DE ACTIVACIÓN DEL EXPANSOR MAXILAR}

Uno de los riesgos asociados es la deglución accidental del instrumental utilizado para la activación del ERM, conocido como llave de activación, que puede ser sólo un alambre o una llave compuesta de un mango más el alambre. Cuando esto ocurre, el instrumento puede transitar por el tracto intestinal sin incidentes hasta ser eliminado en el excremento; o también ser causante de serias complicaciones, ${ }^{(6)}$ tales como: obstrucción de la vía aérea o gastrointestinal, perforación de órganos, hemorragia interna, sepsis o incluso la muerte. ${ }^{(7)}$

La situación siempre debe ser motorizada cercanamente. Si el paciente no reporta sintomatología o padece algún tipo de enfermedad intestinal, se recomienda seguimiento y la eliminación del objeto ocurrirá sin dificultades. Sin embargo, si el objeto en cuestión tiene punta activa y mide más de $5 \mathrm{~cm}$, el riesgo de dañar alguna estructura aumenta considerablemente y debe ser controlado mediante radiografías hasta que se observe la eliminación del artefacto. Si este no se moviliza con normalidad en el intestino, se recomienda removerlo quirúrgicamente. ${ }^{(6)}$ Este riesgo puede ser evitado mediante el uso de adaptadores plásticos para llaves de activación del ERM o asegurandolo con hilo dental. ${ }^{(7)}$

\section{BACTEREMIA TRANSITORIA}

Durante los tratamientos de ortopedia mediante el uso de expansores maxilares, los pacientes son incapaces de mantener una correcta higiene y un efectivo control de la placa bacteriana, lo que genera una inflamación gingival de moderada a severa. En el estudio realizado por Rosa et al., con expansores maxilares de tipo Haas, el 50\% de los pacientes desarrolló una bacteremia transitoria después del retiro del aparato, que se mantiene hasta el quinto día; la colonización correspondía a bacterias Gram +, donde se logró aislar el Streptococcus Oralis. ${ }^{(8)}$ Al mismo tiempo, en el estudio de Gürel et al., se tomaron muestras sanguíneas antes y después del retiro del disyuntor, y se observaron crecimientos bacterianos en un 32\% de las muestras; siendo posible aislar al Streptococcus Viridans en un $16 \%$ de estas. ${ }^{(9)}$

Hay pocas especies bacterianas que son capaces de causar infecciones a distancia una vez que entran al torrente sanguíneo; una de estas es el S. Viridans que es capaz de causar endocarditis bacteriana. Por tanto, en pacientes con riesgo moderado a severo de desarrollar endocarditis bacteriana se debe considerar el uso de profilaxis antibiótica, ${ }^{(9)}$ además de complementar con el uso de enjuagues con clorhexidina en los momentos previos a la remoción del expansor para disminuir los riesgos descritos. ${ }^{(8)}$

\section{Desviación DEL SEPTUM NASAL}

La expansión rápida del maxilar se utiliza para la separación del paladar a través de la sutura media palatina debido a una fuerza lateral del aparato que se apoya en las estructuras dentarias y en los tejidos de soporte. Tanto los huesos cigomáticos como esfenoides de la base craneal generan resistencia durante la expansión. Por lo tanto, la separación de los huesos maxilares se produce de forma triangular, con el vértice hacia la cavidad nasal y la base en el mismo nivel que el proceso palatino lo que resulta en una mayor apertura anterior que posterior. ${ }^{(10)}$

Algunos estudios han informado la corrección de la desviación septal como un hallazgo incidental de ERM. Antiguamente se pensaba que el ERM afectaba la función normal de la vía aérea mediante cambios en el volumen nasal. Sin embargo, en los estudios más 
recientes se ha demostrado que la ERM contribuye a mantener o a enderezar el tabique nasal; a pesar de tener una limitada evidencia disponible, con un riesgo moderado de sesgo, se sugiere un efecto potencialmente positivo sobre la asimetría del tabique nasal durante la infancia, pero no se han encontrado cambios significativos en la adolescencia a partir de la ERM en pacientes con desviación del septum nasal. ${ }^{(10)}$

\section{REABSORCIÓN RADICULAR}

La reabsorción radicular corresponde a una condición patológica en la que el cemento y la dentina radicular se reabsorben. ${ }^{(10)}$ Durante la fase activa de la RME, grandes fuerzas son transmitidas al maxilar, provocando una hialinización del ligamento periodontal, que evita que las piezas dentarias se muevan. Las fuerzas residuales producidas por el aparato, pueden provocar secuelas de la hialinización del ligamento periodontal, tales como la reabsorción radicular. ${ }^{(11)}$

En una revisión bibliográfica realizada por Lo Giudice et al., se evaluó la reabsorción radicular con CBCT luego de la RME, concluyendo que existía una pérdida de volumen radicular en las piezas posteriores, principalmente el primer molar. ${ }^{(11)}$

\section{COMPROMISO PERIODONTAL:}

Durante la terapia de expansión rápida del maxilar, grandes fuerzas son transmitidas al maxilar a través del anclaje de las piezas posteriores, las cuales inducen la hialinización del ligamento periodontal que previene el movimiento dental y busca lograr el efecto terapéutico. Sin embargo, a pesar del beneficio del tratamiento al expandir la sutura palatina, se pueden presentar efectos adversos en los tejidos circundantes y en los mismos dientes, como una inclinación vestibularizada ${ }^{(12)}$ y verticalización ${ }^{(13)}$ de estos mismos a nivel posterior. Otro de los efectos negativos en los tejidos circundantes es la disminución del grosor del hueso alveolar en su pared vestibular y una pérdida de hueso marginal en los dientes que se encuentren anclados al aparato. A nivel periodontal se pueden producir secuelas tales como fenestraciones, dehiscencia, pérdidas de inserción y recesiones gingivales. ${ }^{(12,14)}$

La revisión sistemática realizada por Lo Giudice et al., reporta mediante evaluación con Cone Beam, un adelgazamiento del hueso alveolar en su pared vestibular a nivel de los primeros molares y primeros premolares superiores menor a $1 \mathrm{~mm}$. A nivel del hueso marginal igual se observa una reducción menor de $1 \mathrm{~mm}$. Sin embargo, se debe mencionar que estas evaluaciones fueron realizadas en un corto plazo (no mayor a 6 meses), y no existen estudios a largo plazo que evalúen la recuperación de hueso alveolar y marginal después de la $\mathrm{RME}$. Se recalca que la expansión lenta del maxilar a diferencia de la RME ocasiona pérdidas más graves del hueso alveolar a nivel vestibular. Los siguientes datos se deben tomar en consideración, y evaluar a medida que el tratamiento progresa como reaccionan los tejidos periodontales ante la aplicación de estas grandes fuerzas expansoras. ${ }^{(12)}$

\section{Cambio posicional de los cóndilos}

Además de los cambios en la sutura maxilar, algunos estudios han presentado una asociación entre la expansión maxilar y cambios mandibulares, tales como el aumento del ancho y rotación mandibular. Debido a este movimiento mandibular, se sugiere que los cóndilos presentan un cambio espacial. Muchos estudios han investigado el movimiento de los cóndilos durante la ERM. Dichos estudios indican que la ERM tiene un efecto en la posición de los cóndilos, aunque no han especificado si este efecto es positivo o negativo. ${ }^{(15)}$

No está claro cuál es el efecto directo de ERM en la posición condilar y cómo ocurre. Una hipótesis es que la ERM genera más tipping y dilatación dental que la expansión esquelética, por lo tanto, hace que la mandíbula gire y tenga un contacto posterior prematuro. Así la mandíbula se posiciona más hacia posterior y puede causar presión extra del cóndilo hacia la fosa glenoidea.(15)

\section{CAmbios faciales de teJido BlANDO:}

Uno de los objetivos principales de los ortodoncistas es mejorar la armonía y estética facial mientras logran una oclusión ideal. ${ }^{(16)}$ Huang et al., destacan en su revisión sistemática que, aunque varios estudios han informado sobre los efectos esqueléticos y dentales de la ERM, hay pocos que han abordado las alteraciones de tejido blando. Una de las posibles alteraciones descritas en la literatura son los cambios morfológicos de la nariz. ${ }^{(16)}$

Los hallazgos de Huang et al., sugieren que la ERM genera un aumento del ancho nasal, el ancho de la boca, el ancho del filtrum y la distancia desde el labio inferior hasta la línea $E$ después de un período de observación 
de hasta 6 meses. La mayoría de las mediciones evaluadas mostraron un cambio promedio de $<1 \mathrm{~mm}$, lo que indica una relevancia clínica limitada. Se necesitan más investigaciones, con muestras más grandes y controles apropiados para una evaluación más precisa de las respuestas de los tejidos blandos después de la RME y la estabilidad a largo plazo de estos cambios. ${ }^{(16)}$

\section{Cambios esqueletales}

La literatura señala que inmediato a la ERM se produce un desplazamiento del maxilar hacia abajo y, una extrusión de los dientes de soporte, provocando una rotación mandibular hacia abajo y atrás. Esta rotación produce cambios cefalométricos, como aumentos a nivel de la inclinación del plano mandibular, en la altura facial inferior anterior, en la convexidad facial y también ocasiona una mordida abierta anterior. ${ }^{(17)}$ Sumando a esto, la sobrecorrección de dos a tres milímetros del perímetro del arco maxilar ocasiona interferencias oclusales; ocluyendo las cúspides palatinas de los dientes superiores con las cúspides vestibulares de los dientes mandibulares, contribuyendo a un aumento en la dimensión vertical. ${ }^{(3,17)}$ Es por esto, que algunos ortodoncistas han aconsejado no utilizar la ERM en pacientes con patrones de crecimiento verticales y con perfiles faciales convexos, con el fin de evitar una mayor severidad de la maloclusión. ${ }^{(17)}$

Otro de los riesgos asociados es que la ERM es un procedimiento traumático a la tuberosidad, lo que podría aumentar el riesgo de fractura de ésta, lo que ocasionaría un desplazamiento del maxilar inadecuado hacia abajo y adelante, aunque existe controversia en este punto. ${ }^{(3)}$

\section{DISCUSIÓN}

La expansión rápida del maxilar (ERM) es un procedimiento ortopédico eficaz que se ha utilizado en pacientes en crecimiento durante más de medio siglo. Debido a los variados efectos secundarios positivos en la salud general del paciente, la cantidad de indicaciones para RME ha crecido a lo largo de los años. A pesar de que esta terapia se lleva a cabo para corregir las discrepancias transversales dentales y esqueléticas maxilares, algunos investigadores han demostrado que los resultados del tratamiento también influyen en el aumento de las dimensiones de la vía aérea nasofaríngea, lo que conlleva a una mejor respiración nasal. (18) Sin embargo, no son solo efectos positivos los que se atribuyen a esta terapia, algunos autores, aunque escasos, han descrito algunos efectos negativos, que fueron en los que enfocamos esta revisión.

Dentro de los efectos adversos reportados en la literatura encontramos efectos inherentes a la técnica, y otros prevenibles, tales como la bacteremia transitoria y la deglución accidental del dispositivo de activación del expansor. ${ }^{(6,7,9)}$ El profesional debe ser capaz de realizar una completa anamnesis para tener identificados a los pacientes de alto riesgo de desarrollar endocarditis bacteriana al utilizar la aparatología de ERM y de poder actuar preventivamente, utilizando profilaxis antibiótica. ${ }^{(8,9)}$

La escasa bibliografía encontrada no permite realizar un buen análisis estadístico, debido a la heterogeneidad de los estudios, se hace más complejo la tarea de comparar las diferentes investigaciones. La diversidad de estas en tamaño y edad de la muestra, aparatos utilizados y evaluación de los efectos adversos, no nos permite establecer claramente los riesgos comprobados de la utilización de esta terapia. Además, ningún estudio contaba con controles a largo plazo.

Debemos ser capaces de prevenir de manera efectiva la severidad de la anomalía transversal, ya que ésta se verá acentuada cuando el paciente se encuentre en su peak puberal, y nos veremos enfrentados a considerar como opción terapéutica realizar una ERM asistida quirúrgicamente, ${ }^{(19)}$ la cual no está exenta de efectos secundarios, tales como: dolor, infección, hematomas, sangrado excesivo, necrosis tisular, expansión maxilar asimétrica, recesión gingival, pérdida de hueso periodontal, oscurecimiento y pérdida de dientes. ${ }^{(20)}$

\section{CONCLUSIÓN}

Se puede concluir que la ERM es un procedimiento ortopédico eficaz, sus indicaciones en el tratamiento precoz han aumentado en el tiempo debido a sus resultados positivos. De acuerdo con la bibliografía encontrada los beneficios superan considerablemente a los efectos negativos asociados.

\section{BIBLIOGRAFIA}

1. Majid ZS, Abidia RF. Effects of Malocclusion on oral health related quality of life (OHRQoL): A critical Review. European Scientific Journal. 2015;11(21): 386-400. 
2. Ministerio de Salud, Gobierno de Chile. Diagnóstico Nacional de Salud Bucal del adolescente de 12 años y evaluación del grado de cumplimiento de los objetivos sanitarios de Salud Bucal 2000-2010. Chile. 2007:1-15.

3. Chang JY, Mc Namara JA, Herberger TA. A longitudinal study of skeletal side effects induced by rapid maxillary expansion. Am J Orthod Dentofacial Orthop. 1997; 112(3):330-7

4. Mata J, Zambrano F, Quiros O, Maza P, D Jurisic A, Alcedo C, Fuenmayor D, Ortiz M. Expansión rápida del maxilar en maloclusiones transversales: Revisión bibliográfica. Revista Latinoamericana de Ortodoncia y Odontopediatría. 2009. Obtenible en: https:// www.ortodoncia.ws/publicaciones/2009/art-24/

5. Gregoret J, Tuber E, Escobar H. Aparatología Auxiliar.. El tratamiento Ortodóncico con Arco Recto. Primera edición. Madrid: NM Ediciones; 2003. 281-288.

6. Monini AC, Maia LG, Jacob HB, Gandini LG. Accidental swallowing of orthodontic expansion appliance key. Am J Orthod Dentofacial Orthop. 2011;140(2):266-8.

7. Pantuzo MCG, Nunes E, Pries LR, Pinto LS da MC, Oliveira DD. Ingestion of RPE Activation Key: Why do these accidents still happen?. Eu Arch Paediatr Dent. 2017; 18(2):119-121.

8. Rosa EA, Rached RN, Tanaka O, Fronza F, Fronza F, Araújo Assad R. Preliminary investigation of bacteremia incidence after removal of the Haas palatal expander. Am J Orthod Dentofacial Orthop. 2005;127(1):64-6.

9. Gürel HG, Basciftci FA, Arslan U. Transient bacteremia after removal of a bonded maxillary expansion appliance. Am J Orthod Dentofacial Orthop. 2009;135(2):190-3.

10. Aziz T, Ansari K, Lagravere M, Major M, Flores-Mir C. Effect of non-surgical maxillary expansion on the nasal septum deviation: a systematic review. Progress in Orthodontics. 2015;16(15):1-7.

11. Lo Giudice A, Galletti C, Gay-Escoda C, Leonardi R. CBCT assesment of radicular volume loss after rapid maxillary expansion: A systematic review. J Clin Exp Dent. 2018;10(5):484-494.
12. Lo Giudice A, Barbato E, Cosentino L, Ferraro CM, Leonardi R. Alveolar bone changes after rapid maxillary expansion with tooth-born appliances: a systematic review. Eur J Orthod. 2018; 40(3):296-303.

13. Lione R, Franchi L, Cozza P. Does rapid maxillary expansion induce adverse effects in growing subjects?. Angle Orthod. 2013; 83(1):172-182.

14. Rungcharassaeng K, Caruso J, Kan JYK, Kim J, Taylor G. Factors affecting buccal bone changes of maxillary posterior teeth after rapid maxillary expansion. Am J Orthod Dentofacial Orthop. 2007;132(4): 428.e1-428.e8.

15. McLeod, L., Hernández, I., Heo, G. and Lagravère, $M$. Condylar positional changes in rapid maxillary expansion assessed with cone-beam computer tomography. International Orthodontics. 2016; 14(3): 342-356.

16. Huang, J., Li, C. and Jiang, J. Facial soft tissue changes after nonsurgical rapid maxillary expansion: a systematic review and meta-analysis. Head E Face Medicine. 2018; 14(1)1-10.

17. Garib D, Henriques J, Carvalho P, Gomes S. Longitudinal Effects of Rapid Maxillary Expansion. The Angle Orthodontist. 2007; 77(3):442-448.

18. McNamara, J., Lione, R., Franchi, L., Angelieri, F., Cevidanes, L., Darendeliler, M. and Cozza, P. The role of rapid maxillary expansion in the promotion of oral and general health. Progress in Orthodontics. 2015; 16(1)1-7.

19. Chrcanovic, B. and Custodio, A. Orthodontic or surgically assisted rapid maxillary expansion. Oral and Maxillofacial Surgery. 2009; 13(3): 123-137.

20. Pereira, M., Koga, A., Prado, G. and Ferreira, L. Complications from surgically assisted rapid maxillary expansion with Haas and Hyrax expanders. Journal of Craniofacial Surgery. 2018; 29(2): 275-278.

\section{AUTOR DE CORRESPONDENCIA:}

Autor de correspondencia:

Antonieta Pérez-Flores

Av. Roosvelt 1550, Concepción, Chile

Fono +56998838301

E-mail: mperezf@gmail.com 